\title{
Successful Treatment of Diffuse Large B-cell Lymphoma Presenting as Acute Liver Failure
}

\author{
Rao RL ${ }^{1 *}$, Hornstein $\mathbf{N}^{1}$, Britten $\mathrm{K}^{1}$, Ghafour $\mathrm{SN}^{2}$, \\ Pilley $\mathrm{J}^{3}$ and Rettig $\mathrm{M}^{4}$ \\ ${ }^{1}$ Department of Medicine, University of California Los \\ Angeles (UCLA) Medical Center, USA \\ ${ }^{2}$ Department of Medicine, Division of Hematology- \\ Oncology, University of California Los Angeles (UCLA) \\ Medical Center, USA \\ ${ }^{3}$ Department of Pathology, Veterans Affairs of Greater Los \\ Angeles Health Care System, USA \\ ${ }^{4}$ Department of Urology, University of California Los \\ Angeles (UCLA) Medical Center, USA \\ *Corresponding author: Rao RL, Department of \\ Medicine; University of California Los Angeles (UCLA) \\ Medical Center; 757 Westwood Plaza, Los Angeles, CA \\ 90095 USA
}

Received: May 18, 2021; Accepted: J une 04, 2021; Published: June 11, 2021

\begin{abstract}
Diffuse Large B-Cell Lymphoma (DLBCL) is the most common histologic subtype of lymphoma. Affected patients most commonly present with rapid lymph node enlargement in the neck, abdomen, or chest, with extranodal disease occurring in up to $40 \%$ of all cases. Generally, DLBCL is a clinically aggressive malignancy and the presence of extranodal disease is a poor prognostic indicator. Acute Liver Failure (ALF) is a rare presentation of extranodal DLBCL that is typically associated with a bleak prognosis; however, the impact of prompt diagnosis followed by initiation of appropriate chemotherapy in this setting is not well understood. Further complicating treatment, first-line chemotherapy regimens for DLBCL are typically contraindicated in the setting of liver failure. In this case report, we describe the successful treatment of DLBCL originally presenting as ALF through bridging therapy with a non-standard bendamustinebased approach.
\end{abstract}

Keywords: Diffuse large B-cell lymphoma; Extranodal lymphoma; Acute liver failure; Rituximab; Bendamustine

\section{Case Presentation}

Diffuse Large B-Cell Lymphoma (DLBCL) is the most common histologic subtype of lymphoma, accounting for approximately $25 \%$ of all Non-Hodgkin's Lymphoma (NHL) cases in the developed world [1]. DLBCL is itself a heterogenous entity comprised of several distinct subtypes [2]. Generally, DLBCL is a clinically aggressive malignancy and the presence of extranodal disease is a poor prognostic indicator [3]. Affected patients most commonly present with rapid lymph node enlargement in the neck, abdomen, or chest, with extranodal disease occurring in up to $40 \%$ of all cases [3]. The gastrointestinal tract is the most common extranodal site, though the disease can arise in virtually any tissue in the body. While malignant infiltration by hematological malignancies can cause hepatic dysfunction, this is typically in later stages of the disease [4]. In contrast, Acute Liver Failure (ALF), defined as the development of severe acute liver injury with impaired synthetic function in a patient without preexisting liver disease, is a rare presentation of DLBCL [5-10].

ALF due to malignant infiltration is typically associated with a bleak prognosis with mortality rates as high as $94 \%$ and median time to death of 6 days, according to one case series [11]. However, the impact of prompt diagnosis followed by initiation of appropriate chemotherapy on prognosis is not well understood [12]. Further complicating treatment, first-line chemotherapy regimens for DLBCL are contraindicated in the setting of liver failure. In this case report, we describe the successful treatment of DLBCL which originally presented as ALF through bridging therapy with a non-standard bendamustine-based approach.

We report a case of a 65-year-old African American male with metastatic prostate cancer treated with radiation therapy, leupron and abiraterone, who presented with abdominal pain, nausea and vomiting. His labs were notable for total bilirubin $4.61 \mathrm{mg} / \mathrm{dl}$ (Ref:
0.1-1.2 mg/dl), alkaline phosphatase 728 IU/L (Ref: 44-147 IU/L), ALT 140 U/L (Ref: 7-56 U/L), AST 166 U/L (Ref: 10-40 U/L), total protein $5.1 \mathrm{~g} / \mathrm{dl}$ (Ref: $6-8 \mathrm{~g} / \mathrm{dl}$ ), albumin $3.3 \mathrm{~g} / \mathrm{dl}$ (Ref: 3.4-5.4 g/dl), INR 1.25 (Ref: <1.1), ammonia level $114 \mathrm{u} / \mathrm{dl}$ (Ref: 15-45 u/dl), and LDH 1061 U/L (Ref: 140-280 U/L). Acute hepatitis panel and APAP level were negative.

Despite holding the abiraterone given concern for druginduced liver injury, his bilirubin, liver enzymes and synthetic function progressively worsened. Computed Tomography (CT) of the abdomen and pelvis with contrast demonstrated enlarged liver with heterogeneous enhancement throughout both liver and spleen, along with multiple new enlarged intra-abdominal lymph nodes. Peripheral blood smear was notable for Pseudo-Pelger-Huët neutrophils, toxic granulations and neutrophil precursor band cells. Liver biopsy was performed given rapidly progressive liver failure. Pathology demonstrated large lymphoma cells infiltrating the portal tract (Figure 1), with immunohistochemical staining consistent with aggressive "double expresser" DLBCL, and Ki67 75\%. Fluorescence In Situ Hybridization (FISH) analysis confirmed the diagnosis of high-grade B-cell lymphoma with myc and bcl-6 rearrangement or “double hit" lymphoma (Figure 2).

A staging PET/CT showed diffusely enlarged hypermetabolic lymph nodes with maximum SUV of 10.5 in the neck, axilla, mammary and peri-hepatic nodes as well as hypermetabolism within the porta hepatitis and the right upper lobe.

Given the patient's deteriorating hepatic function, development of encephalopathy, and worsening synthetic function, a decision was made to initiate systemic chemotherapy. Although firstline treatment with R-CHOP (rituximab, cyclophosphamide, doxorubicin, vincristine, and prednisone) was considered, his peak total bilirubin $20.6 \mathrm{mg} / \mathrm{dl}$ (Ref: 0.1-1.2 mg/dl) precluded its use and 


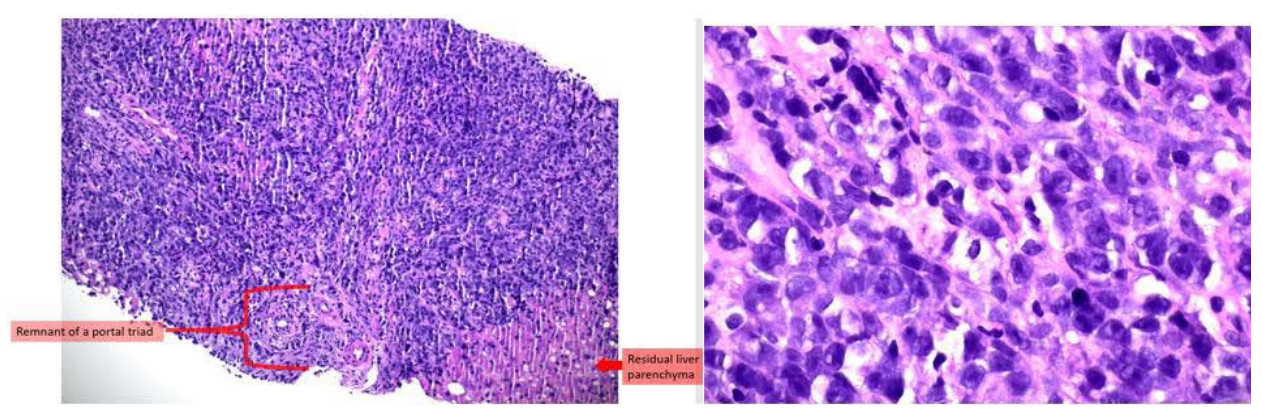

Figure 1: Pathology demonstrating large lymphoma cells with central nucleoli, irregular nuclear contours, and vesicular chromatic. Occasionally, the large lymphoma cells exhibit multiple nucleoli.

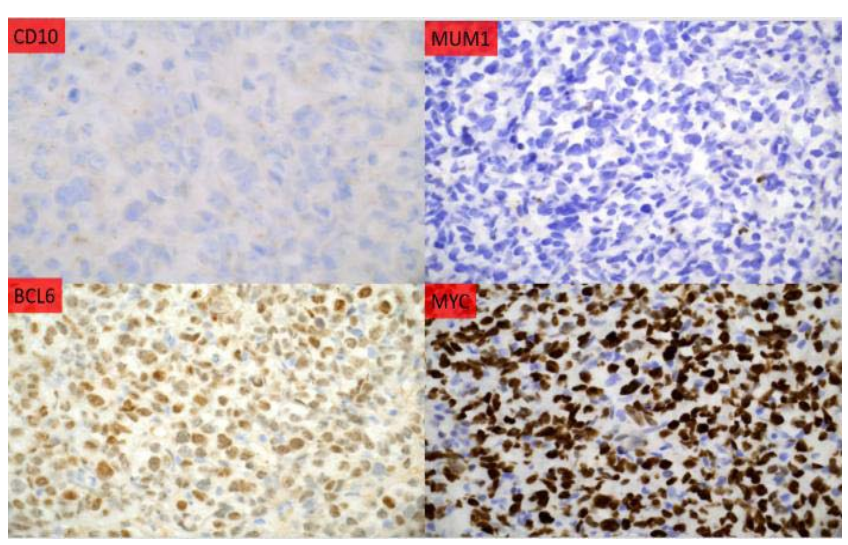

Figure 2: Immunohistochemistry demonstrating CD10, BCL6, MYC and MUM1 expression. In this case the lymphoma cells express a germinal center B-cell like immunophenotype with CD10 (-), BCL6 (+), and MUM1 $(-)$. Additionally, MYC is positive making this lymphoma a Double Expresser which is a poor prognostic indicator. Both BCL6 and MYC were rearranged on FISH analysis which is considered a "Double Hit" and is a specific diagnosis in the WHO, High-grade B-cell lymphoma, Double Hit or High-Grade B-Cell lymphoma with MYC and BCL6 rearrangement.

a less systemically toxic regimen consisting of prednisone, rituximab and bendamustine was chosen for induction. Shortly after initiation of this regimen, there was a rapid and dramatic improvement in both his liver enzymes and markers of synthetic function, with total bilirubin decreased to $7.5 \mathrm{mg} / \mathrm{dl}$, complete normalization of AST/ALT and INR, and resolution of both encephalopathy and presenting gastrointestinal symptoms. Given his rapid functional and clinical improvement, the patient was discharged with a plan to continue therapy with either R-CHOP (rituximab, doxorubicin, cyclophosphamide, vincristine and dexamethasone) or R-DHAP (rituximab, cisplatin, high dose cytarabine and dexamethasone).

However, 2 weeks after initial treatment with prednisone, rituximab and bendamustine, he had recurrent nausea, vomiting and abdominal pain, and was found to have again increasing total bilirubin to $13.0 \mathrm{mg} / \mathrm{dl}$. Given presumed treatment failure, he was challenged with the R-DHAP regimen, which again led to dramatic improvement in liver enzymes (total bilirubin $6.5 \mathrm{mg} / \mathrm{dl}$ ) and overall symptoms.

Three weeks after discharge, he was incidentally found to have worsening total bilirubin to $11.0 \mathrm{mg} / \mathrm{dl}$, prompting admission for treatment with standard first-line R-EPOCH (rituximab, etoposide,

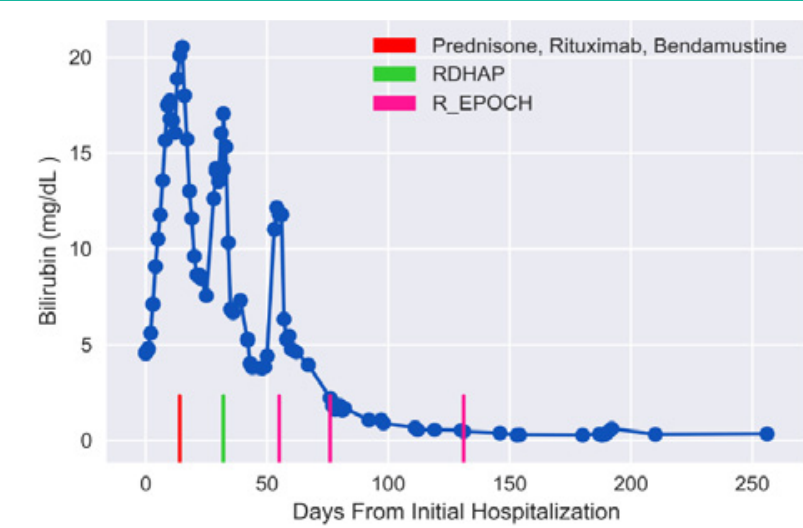

Figure 3: Trends in hepatic function as measured by bilirubin levels. Following challenge with $\mathrm{R}-\mathrm{EPOCH}$, bilirubin levels have been within normal limits.

prednisone, vincristine, cyclophosphamide, and doxorubicin), but with $25 \%$ dose reduction in doxorubicin. He also received prophylactic intrathecal methotrexate. He tolerated the regimen well and received his second cycle of R-EPOCH without incident. PET/ CT after cycle 2 showed significant reduction in previously seen adenopathy and resolution of hypermetabolism. He completed 2 more cycles of R-EPOCH and his liver function returned to normal by end of treatment (Figure 3).

\section{Discussion}

ALF portends a dismal prognosis in the setting of the more common etiologies, but in the context of an underlying double-hit lymphoma, outcomes are even worse. Through prompt recognition, clinical acumen, and selection of appropriate chemotherapy, this life-threatening entity can be treated. This case demonstrates improvements in prognosis with early recognition and treatment, as well as bridging therapies available that can be implemented for patients with ALF secondary to aggressive B-cell lymphomas.

Selection of appropriate treatment regimens for patients with abnormal liver function can be a challenging endeavor. Although many chemotherapies carry a risk of direct hepatotoxicity, it is unclear how drug kinetics, metabolism and systemic toxicities could be affected by liver dysfunction as a majority of clinical trials exclude these patients [12]. As a consequence, many regimens carry direct contraindications for use in the setting of even minor LFT abnormalities. R-CHOP, or R-EPOCH in the case of high grade B-cell 
lymphoma with myc and bcl 2 and/or bcl6 rearrangements, remains the standard of care for treatment of DLBCL $[13,14]$. In this case, the patient's grossly abnormal LFTs, diminished synthetic function, and hepatic encephalopathy precluded him from receiving the front-line therapy for his aggressive B-cell lymphoma.

Bendamustine, a fairly well tolerated chemotherapy, has historically been deployed in the context of mantle cell and indolent lymphomas as well as in salvage therapy for relapsed or refractory DLBCL [15-18]. Although the pharmacokinetics and safely profile of Bendamustine in the setting of liver failure are not well understood, its tolerability and use in several forms of lymphoma makes it a promising agent if standard treatments cannot be used. Additionally, evidence suggests Bendamustine may be tolerable in patients with liver failure $[19,20]$.

Due to the patient's liver failure, use of R-EPOCH was precluded at the time of his presentation and Bendamustine was utilized as a bridge treatment which later allowed for initiation of more definitive therapy with R-DHAP followed by R-EPOCH once his liver synthetic function and hepatic encephalopathy improved. This report joins several others to demonstrate the possible utility of Bendamustine as a bridge therapy in patients with DLBCL who present in the setting of ALF [19,20].

Although clinical trials would provide the gold standard of evidence for the safety and efficacy of therapy in the setting of ALF, the impracticality of such a study necessitates the use of clinical judgement and case reports to help guide treatment in this unique situation. In this case, liver function improved dramatically following the first cycle of Bendamustine, Rituximab and Prednisone. Although this treatment did not provide a prolonged response, it allowed the patient to survive until he could be transitioned to the standard of care with R-EPOCH. This case study illustrates that prompt initiation of a non-standard Bendamustine containing regimen in acute liver failure caused by DLBCL may safely and effectively act as bridge to improve liver function enough to allow initiation of standard regimens that may have the potential to reduce the historically high mortality rate of malignancy associated ALF.

\section{Clinical Practice Points}

Diffuse Large B-Cell Lymphoma (DLBCL) is the most common histologic subtype of lymphoma. Affected patients most commonly present with rapid lymph node enlargement in the neck, abdomen, or chest, with extranodal disease occurring in up to $40 \%$ of all cases. Generally, DLBCL is a clinically aggressive malignancy and the presence of extranodal disease is a poor prognostic indicator. Acute Liver Failure (ALF) is a rare presentation of extranodal DLBCL that is typically associated with a bleak prognosis; however, the impact of prompt diagnosis followed by initiation of appropriate chemotherapy in this setting is not well understood. Further complicating treatment, first-line chemotherapy regimens for DLBCL are typically contraindicated in the setting of liver failure. In this case report, we describe the successful treatment of DLBCL originally presenting as ALF through bridging therapy with a nonstandard bendamustine-based approach. In this case, the patient's grossly abnormal LFTs, diminished synthetic function, and hepatic encephalopathy precluded him from receiving the front-line therapy for his aggressive B-cell lymphoma. Bendamustine was utilized as a bridge treatment which later allowed for initiation of more definitive therapy with R-DHAP followed by R-EPOCH once his liver synthetic function and hepatic encephalopathy improved. This case study illustrates that prompt initiation of a non-standard Bendamustine containing regimen in acute liver failure caused by DLBCL may safely and effectively act as bridge to improve liver function enough to allow initiation of standard regimens that may have the potential to reduce the historically high mortality rate of malignancy associated ALF.

\section{References}

1. Han X, Kilfoy B, Zheng T, et al. Lymphoma survival patterns by WHO subtype in the United States, 1973-2003. Cancer Causes \& Control. 2008; 19: 841858.

2. Swerdlow SH, Campo E, Pileri SA, et al. The 2016 revision of the World Health Organization classification of lymphoid neoplasms [Internet]. Blood. 2016; 127: 2375-2390.

3. Moller MB, Pedersen NT, Christensen BE. Diffuse large B-cell lymphoma: clinical implications of extranodal versus nodal presentation - a populationbased study of 1575 cases. British Journal of Haematology. 2004; 124: 151-159.

4. Risdall R, Hoppe RT, Warnke R. Non-Hodgkin's lymphoma: a study of the evolution of the disease based upon 92 autopsied cases. Cancer 1979; 44: $529-542$.

5. Cervantes F, Briones J, Bruguera M, et al. Hodgkin's disease presenting as a cholestatic febrile illness: incidence and main characteristics in a series of 421 patients. Annals of Hematology. 1996; 72: 357-360.

6. Fairbank WH. Three atypical cases of Hodgkin's Disease, presenting with liver failure. Can Med Assoc J 1953; 69: 315-317.

7. Chim CS, Choy C, Ooi CG, Liang R. Hodgkin's disease with primary manifestation in the liver. Leuk Lymphoma 2000; 37: 629-632.

8. Lettieri CJ, Berg BW. Clinical features of non-Hodgkins lymphoma presenting with acute liver failure: a report of five cases and review of published experience. Am J Gastroenterol. 2003; 98: 1641-16946.

9. Rowbotham $D$, Wendon J, Williams R. Acute liver failure secondary to hepatic infiltration: a single centre experience of 18 cases. Gut 1998; 42: 576-580.

10. Ma Y-J, Chen E-Q, Chen X-B, Wang J, Tang H. Primary hepatic diffuse large B cell lymphoma: A case report: Primary hepatic diffuse large B cell lymphoma. Hepat Mon 2011; 11: 203-205.

11. Floyd J, Mirza I, Sachs B, Perry MC. Hepatotoxicity of Chemotherapy. Seminars in Oncology. 2006; 33: 50-67.

12. Armitage JO. How I treat patients with diffuse large B-cell lymphoma. Blood. 2007; 110: 29-36.

13. Friedberg JW. How I treat double-hit lymphoma. Blood. 2017; 130: 590-596.

14. Rummel MJ, Maschmeyer G, Ganser A, et al. Bendamustine Plus Rituximab (B-R) Versus CHOP Plus Rituximab (CHOP-R) As First-Line Treatment in Patients with Indolent and Mantle Cell Lymphomas (MCL) - 7 Year Updated Results from the StiL NHL1 Study [Internet]. Blood. 2014; 124: 4407-4407.

15. Friedberg JW, Cohen $\mathrm{P}$, Chen $\mathrm{L}$, et al. Bendamustine in patients with rituximab-refractory indolent and transformed non-Hodgkin's lymphoma: results from a phase II multicenter, single-agent study. J Clin Oncol. 2008; 26: 204-210.

16. Weidmann E, Kim S-Z, Rost A, et al. Bendamustine is effective in relapsed or refractory aggressive non-Hodgkin's lymphoma. Ann Oncol. 2002; 13: 1285-1289.

17. Ogura M, Ando K, Niitsu N, et al. A multicenter phase II study of bendamustine with rituximab in patients with relapsed/refractory diffuse large B-cell lymphoma (DLBCL) [Internet]. Journal of Clinical Oncology. 2012; 30: 8023-8023. 
18. Rituximab Plus Bendamustine for the Treatment of Aggressive Non-Hodgkin Lymphoma Patients With Severe Liver Impairment - Hematology \& Oncology. 2020.

19. Schoppmeyer K, Kreth F, Wiedmann M, Mössner J, Preiss R, Caca K. A pilot study of bendamustine in advanced bile duct cancer. Anticancer Drugs. 2007; 18: $697-702$
20. Rummel MJ, Niederle N, Maschmeyer G, et al. Bendamustine plus rituximab (B-R) versus $\mathrm{CHOP}$ plus rituximab (CHOP-R) as first-line treatment in patients with indolent and Mantle Cell Lymphomas (MCL): Updated results from the StiL NHL1 study [Internet]. Journal of Clinical Oncology. 2012; 30: 3-3. 